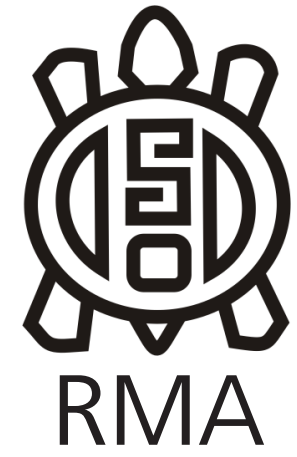

Dossier

\title{
Cuarzo y paisajes productivos en el Cerro Ampuqcatao (Valle de Tafí, Tucumán) durante el segundo milenio D.C. Aplicación de los Métodos M.A.N.A. y No Tipológico
}

\author{
Quartz and productive Landscapes in Ampuqcatao hill (Tafí Valley, \\ Tucumán Province) during second millennium. Application of M.A.N.A \\ and Non Typological methods
}

Juan M. Montegú*

*Departamento de Geología, Universidad Nacional de San Luis. CONICET. E-mail: juanmontegu@unc.edu.ar

\section{Resumen}

Se exponen los resultados de la aplicación de los métodos M.A.N.A. y No Tipológico a conjuntos líticos de cuarzo, recuperados en el sitio arqueológico El Alto, ubicado en el sector norte del cerro Ampuqcatao (Valle de Tafí, Tucumán). Los materiales provienen de la excavación de dos recintos asociados a terrazas de cultivo que conforman un conjunto productivo adscribible al segundo milenio D.C. El estudio apuntó a reconocer las etapas de la secuencia productiva lítica que se desarrollaron en los recintos, comprender las prácticas en las que se relacionaron materiales, personas y paisaje, y determinar la potencialidad de las metodologías para el estudio de artefactos de cuarzo. Los resultados permitieron identificar las etapas iniciales de la producción lítica, las que se habrían llevado a cabo en el marco de estrategias embedded vinculadas a tareas de producción agropastoril. Por otra parte, las metodologías mostraron ser complementarias con estudios tecno-tipológicos, permitiendo una mejor comprensión de las secuencias tecnológicas líticas.

Palabras clave: Cuarzo; Valle de Tafí; Paisajes productivos; M.A.N.A.; No tipológico.

\begin{abstract}
We present the results of M.A.N.A. and Non Typological methods applied quartz lithic assemblages recovered at EI Alto archaeological site, located in the north side of Ampuqcatao hill (Tafi Valley, Tucumán Province). The materials came from the excavation of two enclosures associated to farming terraces which forms a productive set ascribed to the second millennium. The study was aimed at recognizing stages of lithic production sequence developed within the enclosures, to understand the practices that would have related materials, people and landscape, and to determine the potential of the methodologies for the study of quartz artifacts. The results allowed identifying early stages of lithic production, which would be carried out within embedded strategies related to agropastoral production tasks. On the other hand, methodologies proved to be complementary of techno-typological studies allowing a better understanding of the lithic technological sequences.
\end{abstract}

Keywords: Quartz; Tafí Valley; Productive landscape; M.A.N.A.; Non typological.

\section{Introducción}

El cuarzo forma parte integral del sistema orográfico de las Sierras Pampeanas Noroccidentales en general (Servicio Geológico Argentino 2010). Sus características físico-mecánicas y su ubicuidad lo convirtieron en un recurso altamente utilizado por las sociedades prehispánicas que ocuparon el valle de Tafí, lo cual se refleja en el alto porcentaje de dicha materia prima en los conjuntos líticos recuperados en el mismo (Franco Salvi et al. 2016). Sin embargo, diversos investigadores han subrayado las dificultades para su estudio debido a los inconvenientes que se presentan para reconocer características diagnósticas de talla (Cattáneo 1994; Kulemeyer y Laguna 1995; Orquera y Piana 1986; Pautassi 2010). Esta situación propicia la aplicación de metodologías que permitan un análisis más profundo sobre el uso de esta materia prima en diferentes contextos arqueológicos.

El objetivo de este trabajo es reconocer las etapas de la secuencia productiva lítica que se desarrollaron en contextos arqueológicos excavados en el sitio El Alto (Valle de Tafí, Tucumán), los cuales se asocian a una 
cronología relativa asignable al segundo milenio D.C. Para ello se aplicaron los lineamientos de los análisis M.A.N.A. (Larson y Kornfeld 1997) y No tipológico (Ingbar et al. 1989), así como estudios tecno-tipológicos a los conjuntos líticos de cuarzo recuperados. Al contextualizar estos resultados se buscó comprender las prácticas que habrían relacionado a los materiales, las personas y el paisaje circundante, y determinar la potencialidad de las metodologías aplicadas para el estudio de artefactos de cuarzo.

\section{Procedencia de los materiales analizados}

El cerro Ampuqcato (Pelao o Loma del Medio) se localiza en el sector central del valle de Tafí (Tucumán, Argentina) (Figura 1), presenta una altura de 2.600 msnm con cumbres planas de escasa pendiente, laderas pronunciadas y quebradas profundas. Los recursos hídricos son limitados ya que casi ninguno de los cursos de agua es permanente y todos se encuentran en lugares de difícil acceso (Salazar 2014).

Mediante un acuerdo con la Comunidad Indígena del Valle de Tafí, en 2014 se realizaron prospecciones en el sector norte del cerro para identificar y valorizar las evidencias arqueológicas presentes en el mismo. Se relevaron recintos habitacionales de planta circular y rectangular, estructuras productivas circulares y cuadrangulares, terrazas de cultivo, líneas de contención del suelo, conjuntos monticulares de despedres, instrumentos de molienda fijos, rocas grabadas, apachetas y recintos circulares y semicirculares aislados (Salazar 2014). La adscripción temporal del sitio no cuenta hasta el momento con dataciones absolutas, sin embargo se realizaron cronologías relativas en función del diseño de las estructuras: 1) se identificaron unidades con características similares a unidades residenciales datadas en el primer milenio D.C. (Berberián y Nielsen 1988; González y Núñez Regueiro 1960; Salazar 2010; Salazar et al. 2008); 2) se reconocieron diversos espacios que presentaban terrazas lineales paralelas y rocas grabadas con cavidades circulares y surcos que forman "maquetas", asignables ambos rasgos al Periodo de Desarrollo Regionales (Nuñez Regueiro 1974; Scattolin 2006); 3) es posible que las "maquetas" también puedan asociarse a una influencia incaica (Williams et al. 2005); 4) la presencia de algunas estructuras con muros pircados sería asociable a momentos subactuales. Es decir, que el sector norte del cerro Ampuqcatao presenta una larga y persistente ocupación que parece iniciarse a principios del primer milenio D.C. y se continúa hasta la actualidad.

Como parte de las actividades acordadas con la comunidad indígena, se coordinó en el área prospectada la instalación de los Circuitos Arqueológicos Comunitarios en un sector conocido como El Alto. Para ello se efectuaron excavaciones en dos estructuras circulares aisladas emplazadas entre sistemas de terrazas aparentemente de cultivo (Figura 2) con la finalidad de que se integrarán en los circuitos interpretativos y de donde proceden los materiales líticos aquí analizados. A falta de materiales datables o cerámica diagnóstica, y debido a la relación arquitectónica de los recintos y las terrazas inmediatas, las estructuras circulares y los artefactos recuperados fueron asignados al segundo milenio D.C.

Las dos estructuras estudiadas son de morfología circular y se identificaron como Estructura 1 (E1, de 2,10 m de diámetro) y Estructura 2 (E2, de 2,20 m de diámetro). En ambos casos, los recintos se encuentran separados de las terrazas por escasos metros de distancia y se ubican en sectores de alta visibilidad tanto de los aterrazamientos circundantes como de las quebradas del cerro y del sector norte del valle de Tafí1. Arquitectónicamente, las estructuras están conformadas por muros de rocas graníticas de mediana a gran dimensión (de entre 0,50 y 0,90 m de largo), disponibles en el mismo cerro, los cuales determinan lienzos firmes que se cierran hacia la parte superior (a modo de semi-bóveda) y cuyo techo debió estar constituido de material perecedero. En E2 se identificó una abertura de 0,45 mts con jambas a sus lados, probablemente utilizada como puerta de acceso al recinto. Las excavaciones alcanzaron los 0,55 $\mathrm{mts}$ de profundidad en E1 y los 0,75 mts en E2, Ilegando a la roca madre en ambos casos. Si bien cada estructura presentó dos estratos naturales, la ausencia de pisos consolidados y de fogones, y la escasa presencia de material cerámico², permitió analizar los conjuntos líticos de ambas capas en cada estructura como una sola muestra.

\section{Metodologías aplicadas}

Dado que los conjuntos líticos analizados se componen casi en su totalidad de desechos de talla y núcleos de cuarzo, nos propusimos experimentar la aplicación de metodologías alternativas, empleando los Análisis No Tipológico (Ingbar et al. 1989) y de Nódulos Mínimos o M.A.N.A. (por sus siglas en inglés) (Larson y Kornfeld 1997). Estas metodologías se originaron en estudios experimentales de desechos que buscaban construir modelos interpretativos que permitieran: identificar un conjunto mínimo de atributos en cada desecho lo suficientemente objetivo para un análisis intraobservador, desarrollar un enfoque para analizar los conjuntos de desechos a partir del trabajo de un mismo bloque de roca como unidad y emplear modelos con funciones matemáticas que permitan el estudio de

\footnotetext{
1 Panorámicamente, ambas estructuras permiten visibilizar El Abra del Infiernillo, el sector medio y norte de la Sierra del Aconquija (incluido el Cerro Muñoz) y las áreas arqueológicas de Las Carreras, El Tolar y La Bolsa.

${ }^{2}$ En E1 se recuperaron cuatro fragmentos cerámicos y en E2 cinco. Todos los tiestos corresponden a piezas oxidantes gruesas sin baños y en ningún caso se identificaron partes diagnósticas.
} 


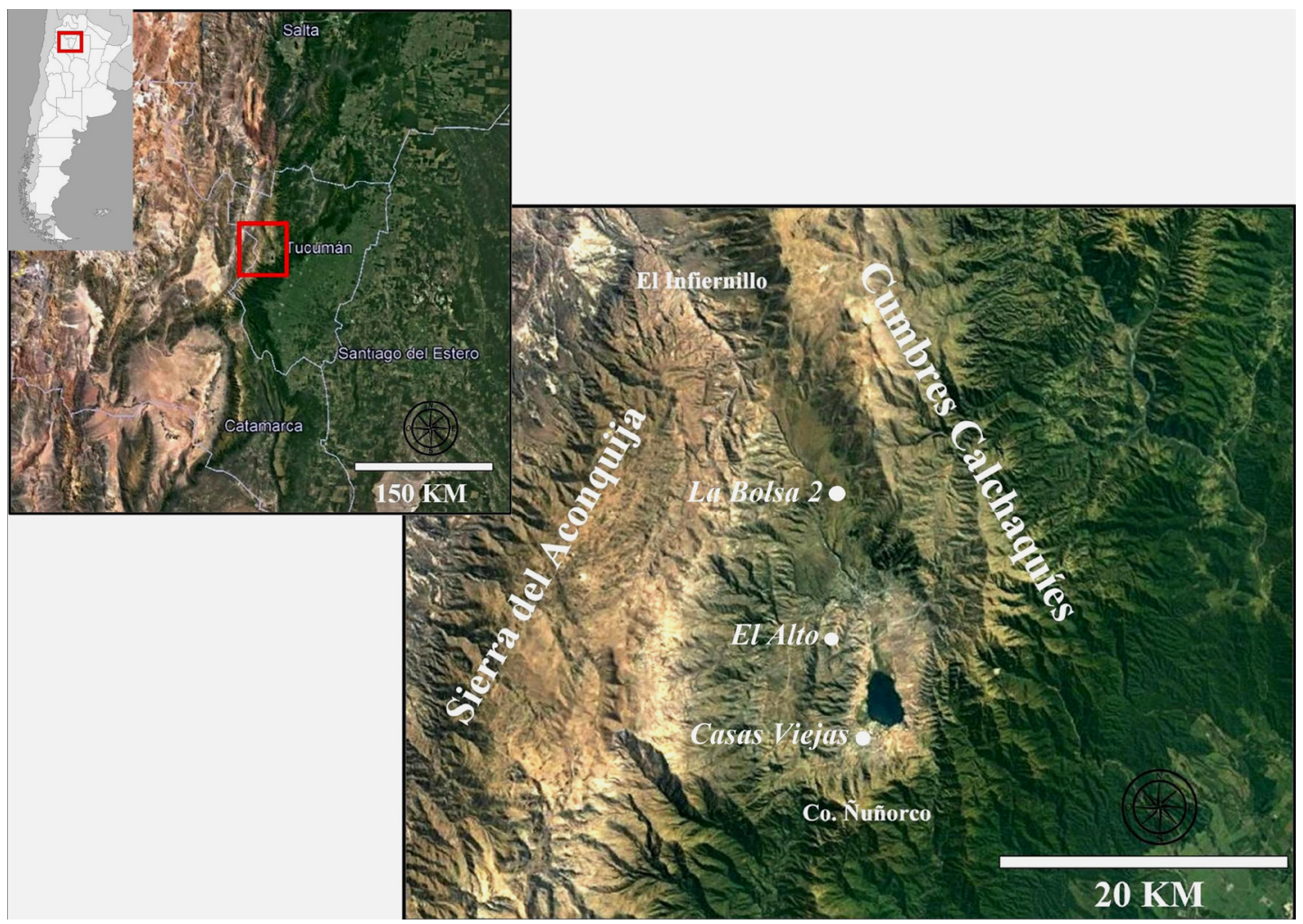

Figura 1. Ubicación del valle de Tafí y sitios mencionados en el texto.

Figure 1. Location of Tafi Valley and sites mentioned in the text.

conjuntos de desechos como parte de un continuum (Flegenheimer y Cattáneo 2013; Ingbar et al. 1989; Larson y Kornfeld 1997).

Hasta el momento, en la literatura arqueológica ambos análisis mencionados se han aplicado a conjuntos líticos de grupos cazadores-recolectores tanto a nivel nacional como internacional (Cattáneo 2002; Charlin 2009; Flegenheimer y Cattáneo 2013; Frison 1974; Kelly 1985; Pautassi 2010; Sario 2011). Sin embargo, se observa en las metodologías una potencialidad para analizar conjuntos líticos provenientes de sociedades productoras de alimentos que manejaron materias primas en las cuales los atributos tipológicos son difíciles de determinar, como en el caso del cuarzo. Los análisis se destacan por ofrecer datos complementarios sobre el proceso de manufactura, las actividades desarrolladas en diversos espacios y la presencia de actores sociales (Pautassi 2010).

En primera instancia los artefactos se agruparon por características macroscópicas de color, tipo de granos e inclusiones. De esta manera se conformaron nódulos mínimos analíticos, que son todos los artefactos que potencialmente proceden de un mismo nódulo. Estos nódulos mínimos fueron clasificados en simples y múltiples. Los nódulos simples están constituidos por un solo artefacto, ya sea un instrumento formatizado, un núcleo o una lasca; los nódulos múltiples son aquellos compuestos por varias piezas, pudiendo combinarse instrumentos, desechos y núcleos (Larson y Kornfeld 1997).

Como segundo paso, entre las lascas enteras de cada nódulo mínimo analítico se registraron los siguientes atributos de carácter ordinal: ancho máximo, largo máximo, espesor en la sección media y cantidad de lascados en la cara dorsal. A estos atributos se les aplicó la fórmula matemática del Modelo $\mathrm{N}^{\circ} 4$ propuesto por Ingbar y colaboradores $(1989)^{3}$. La selección de este modelo se basa en que la curva de regresión que produce es la más real para determinar cuándo se extrajo un desecho en particular. De esta manera, y considerando a la talla como un proceso continuo, se determina el momento en que el desecho habría sido tallado. Finalmente, se realizaron gráficos de dispersión y tablas de promedio de extracción que permitieron observar en cada nódulo mínimo cuál de las etapas del proceso de talla se encuentren representadas, o no, en el conjunto artefactual.

\footnotetext{
3 Predicho $=-12.14 \times(\log E)+9.65 \times(\log D L D)$. Donde: $\log =$ Logaritmo; $\mathrm{E}=$ espesor de la lasca; $\mathrm{DLD}=$ densidad de lascados dorsales (cantidad de negativos de lascados en el dorso/ ancho x largo de la pieza).
} 


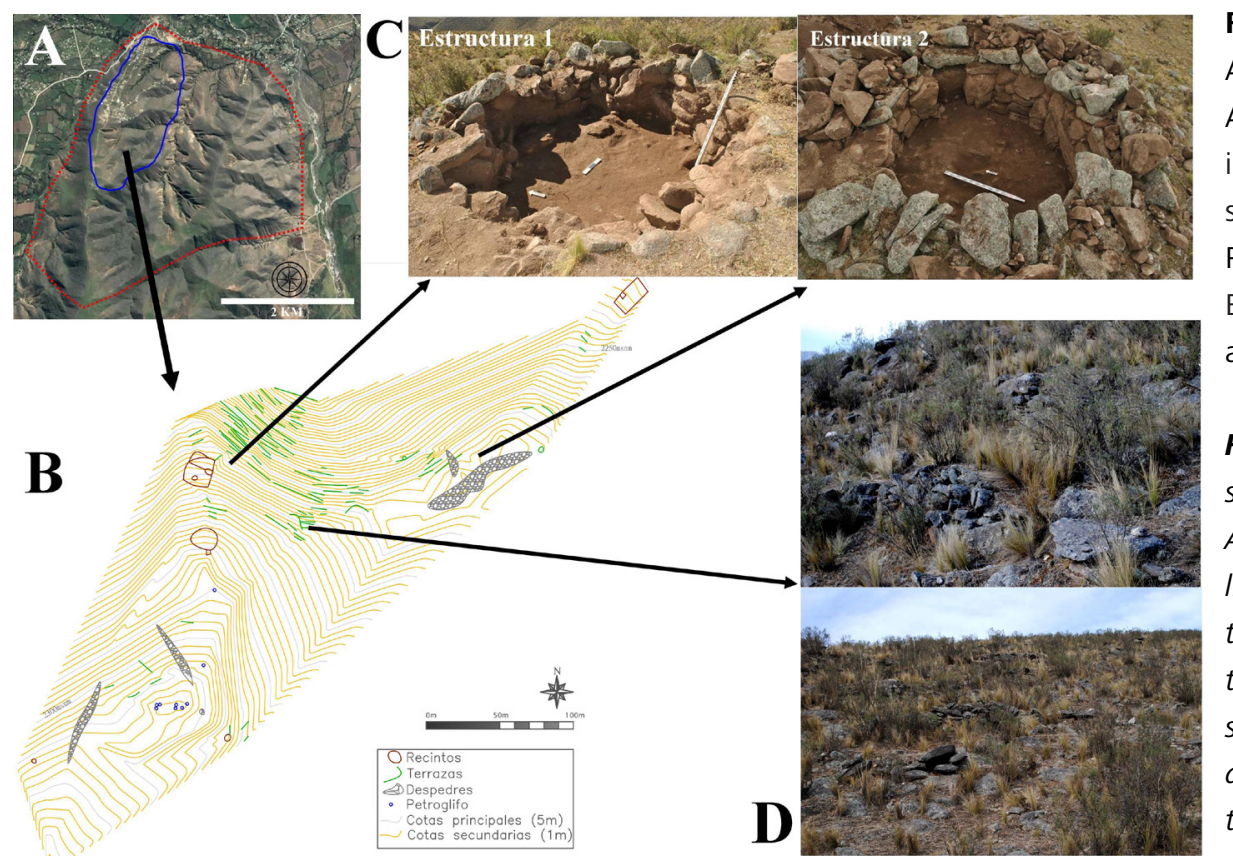

Figura 2. Sitio arqueológico El Alto. A: Sector norte del cerro Ampuqcatao (la línea punteada roja indica el área prospectada y la línea sólida azul los límites del sitio). B: Planta del sector sur del sitio. C: Estructuras E1 y E2. D: Detalle de andenes próximos a E1 y E2.

Figura 2. El Alto archaeological site. A: Northern sector of Ampuqcatao hill (the dotted red line indicates prospected area and the solid blue line boundaries of the site). B: Plant of the southern sector of the site. C: Structures E1 and E2. D: Detail of platforms next to $E 1$ and $E 2$.

Dado que la aplicación de los análisis No Tipológico y M.A.N.A permiten una forma alternativa de acercamiento a las prácticas líticas y que en los conjuntos analizados también se identificaron núcleos, se complementó el estudio con análisis tecno-tipológicos que siguen las propuestas de Aschero (1975; 1983) y de Sullivan y Rozen (1985) adaptadas para el caso de estudio. En el caso de los desechos de talla se registraron como variables: estado de fragmentación, origen de la extracción (lascas internas, externas, de formatización y bipolar) , tamaño, presencia de corteza y tipo de talón. Para los núcleos se observó: estado de la pieza, presencia de corteza, cantidad de lascados y morfología.

\section{Resultados}

Los conjuntos líticos recuperados en las excavaciones de E1 y E2 se componen de 91 y 209 artefactos respectivamente. En ellos, hay una amplia preponderancia del cuarzo, que representa el 98\% $(\mathrm{N}=89)$ en el caso de $\mathrm{E} 1$ y el $99 \%(\mathrm{~N}=206)$ en $\mathrm{E2}$. Los muy escasos porcentajes de las restantes materias primas corresponden a piezas de sílice, rocas graníticas e indeterminadas. Como mencionamos, en este análisis se tomaron en consideración los artefactos de cuarzo. En E1 se identificaron 85 desechos de talla, cuatro núcleos y ningún instrumento, mientras que en E2 se registraron 198 desechos de talla, siete núcleos y un instrumento (Tabla 1).

En base a los lineamientos metodológicos mencionados se pudieron agrupar los materiales cuarzosos en 14 nódulos mínimos para E1 y en 18 para E2 (Tabla 1). La mayoría de estos nódulos corresponden a los tipos múltiples compuestos por más de un desecho de talla (86\% en E1 y $89 \%$ en E2), en tanto que son menos
Tabla 1. Resultados del análisis M.A.N.A. por Estructura. NUC: núcleos; IN: instrumentos; DT: desechos de talla.

Table 1. Results of M.A.N.A. by Structure. NUC: cores; IN: instruments; DT: knapping wastes.

\begin{tabular}{|c|c|c|c|c|c|c|}
\hline Recinto & MANA & NUC & IN & DT & Total & Tipo de Módulo \\
\hline \multirow{15}{*}{$\begin{array}{c}\text { Estructura } \\
1\end{array}$} & 1 & & & 11 & 11 & Múltiple \\
\hline & 2 & & & 4 & 4 & Múltiple \\
\hline & 3 & & & 4 & 4 & Múltiple \\
\hline & 4 & 1 & & 5 & 6 & Múltiple \\
\hline & 5 & & & 2 & 2 & Múltiple \\
\hline & 6 & & & 2 & 2 & Múltiple \\
\hline & 7 & & & 1 & 1 & Simple \\
\hline & 8 & & & 8 & 8 & Múltiple \\
\hline & 9 & 1 & & 7 & 8 & Múltiple \\
\hline & 10 & 1 & & 13 & 14 & Múltiple \\
\hline & 11 & 1 & & 5 & 6 & Múltiple \\
\hline & 12 & & & 20 & 20 & Múltiple \\
\hline & 13 & & & 2 & 2 & Múltiple \\
\hline & 14 & & & 1 & 1 & Simple \\
\hline & Total & 4 & 0 & 85 & 89 & \\
\hline \multirow{19}{*}{$\begin{array}{c}\text { Estructura } \\
2\end{array}$} & 1 & 1 & & 8 & 9 & Múltiple \\
\hline & 2 & 1 & & 15 & 16 & Múltiple \\
\hline & 3 & 1 & & 8 & 9 & Múltiple \\
\hline & 4 & 1 & & 6 & 7 & Múltiple \\
\hline & 5 & & & 5 & 5 & Múltiple \\
\hline & 6 & 1 & & 6 & 7 & Múltiple \\
\hline & 7 & & & 11 & 11 & Múltiple \\
\hline & 8 & & & 2 & 2 & Múltiple \\
\hline & 9 & 1 & & & 1 & Simple \\
\hline & 10 & & & 50 & 50 & Múltiple \\
\hline & 11 & & & 21 & 21 & Múltiple \\
\hline & 12 & & 1 & 23 & 24 & Múltiple \\
\hline & 13 & & & 7 & 7 & Múltiple \\
\hline & 14 & & & 14 & 14 & Múltiple \\
\hline & 15 & 1 & & 4 & 5 & Múltiple \\
\hline & 16 & & & 15 & 15 & Múltiple \\
\hline & 17 & & & 2 & 2 & Múltiple \\
\hline & 18 & & & 1 & 1 & Simple \\
\hline & Total & 7 & 1 & 198 & 206 & \\
\hline
\end{tabular}




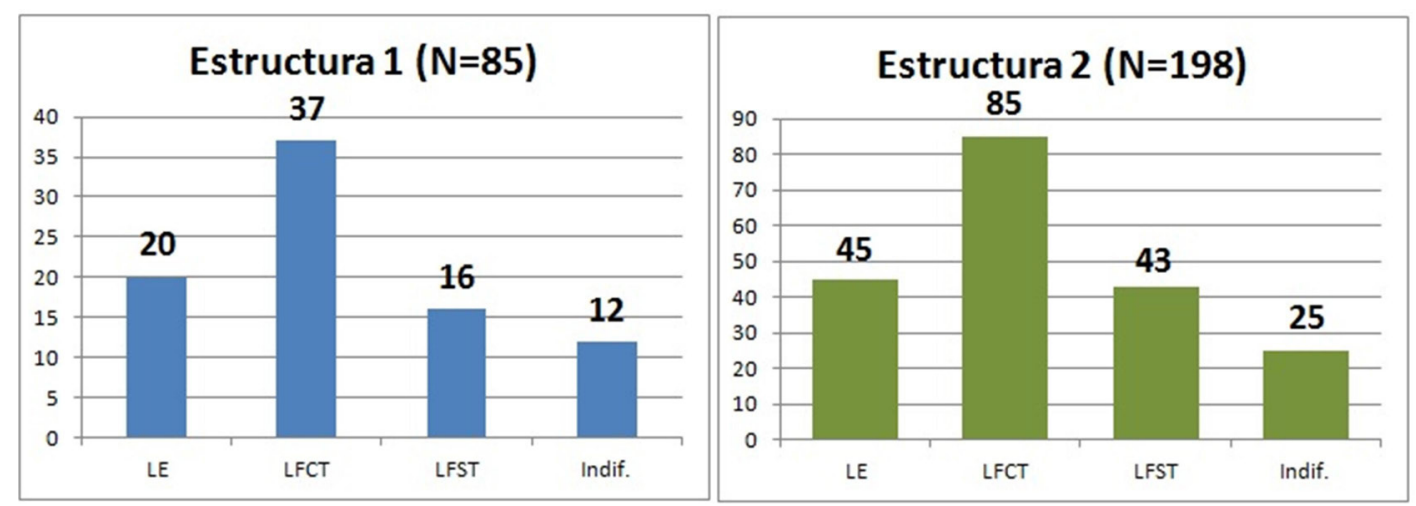

Figura 3. Estado de fragmentación de los desechos de talla. LE: lascas enteras; LFCT: lascas fracturadas con talón; LFST: lascas fracturadas sin talón; Indif.: indiferenciados.

Figure 3. State of fragmentation of knapping wastes. LE: whole flakes; LFCT: fractured flakes with heel; LFST: fractured flakes without heel; Indif:: undifferentiated.

frecuentes los nódulos múltiples formados por desechos y núcleos y/o instrumentos (14\% en E1 y $11 \%$ en E2). Por otra parte, se observaron cuatro nódulos simples, dos de ellos compuestos solo por un desecho de talla en E1 y dos en E2, uno referido a un desecho de talla y el otro a un núcleo.

Al analizar los desechos de talla se observa en la Figura 3 un alto porcentaje de fragmentación, ya que las lascas fracturadas con y sin talón representan el $62 \%$ en la E1 y el 65\% en E2. También es destacable la presencia de lascas enteras ( $24 \%$ en E1 y $23 \%$ en E2) y el bajo porcentaje de desechos indiferenciados (14\% en E1 y $13 \%$ en $\mathrm{E} 2$ ).

Es importante resaltar que el análisis No Tipológico solo puede realizarse sobre las lascas enteras, ya que son estas las que presentan la totalidad de los atributos necesarios para su aplicación. Cada lasca entera de un nódulo mínimo (M.A.N.A) sobre la que se aplica el modelo matemático de Ingbar y colaboradores (1989) se refleja en un grafico de dispersión en donde es representada como un punto que establece el momento específico de su extracción del nódulo. En base a esto, la Figura 4 muestra que en E1 se pudieron identificar nueve eventos distintos de talla para una muestra de 20 desechos. Como se puede observar las extracciones arrancan entre los momentos seis y ocho, y alcanzan hasta los 20 y 21, encontrándose una considerable dispersión entre los nódulos que presentan más de un desecho y la mitad de los eventos con un solo desecho registrado. A su vez, el promedio de momento de extracción para los eventos de talla (Tabla 2) abarca desde el momento 12 al 20.

En el caso de E2 se reconocieron 14 eventos de talla para un conjunto de 45 desechos (Figura 4). Las extracciones se inician entre los momentos uno y seis, llegando hasta los 20 y 22, en tanto que se observa una mayor concentración entre los momentos 10 y 17 . Se destacan los eventos M2, M9, M10 y M11, los cuales incluyen seis $u$ ocho extracciones y muestran una representación más continua de la reducción de los nódulos. En cuanto al promedio de momento de extracción, en E2 se ubican entre el momento uno y 22 (Tabla 2); ahora, si se deja de lado el evento M6 que solo contiene un desecho de talla al inicio de la extracción, el promedio es entre el momento siete y 22 .

Al complementar los análisis anteriores con estudios tecno-tipológicos se obtiene una mirada más amplia de las prácticas de talla efectuadas en las estructuras. En el caso de los desechos de talla, la Tabla 3 muestra un predominio de lascas internas (88\% en E1 y $91 \%$ en E2), ausencia de corteza (70\% en E1 y 62\% en E2) y talones preparados que incluyen los lisos, diedros, facetados y puntiformes (70\% en E1 y $84 \%$ en E2). Respecto a los tamaños, se destaca que existen diferencias entre las estructuras ya que en E1 son preponderantes los desechos de grandes dimensiones -muy grande, grande y mediano grande- (60\%) mientras que en E2 son mayoritarios los pequeños y mediano pequeños (69\%). Además de estas tendencias generales, se debe mencionar la presencia de lascas externas y talones corticales en ambas estructuras, así como el registro de lascas bipolares y talones puntiformes.

Los núcleos identificados (Tabla 4 y Figura 5), considerando ambas estructuras, muestran un alto porcentaje de artefactos agotados y parcialmente agotados $^{4}(73 \%)$, mayoría de piezas sin rastros de

\footnotetext{
4 El cuarzo se presenta en afloramientos relativamente cercanos a las estructuras, los cuales se componen de bloques a modo de clastos en la formación granítica del cerro Ampuqcatao. Las dimensiones de los bloques promedian los $15 \times 15 \mathrm{~cm}$.
} 
corteza (56\%) y una alta variabilidad en las morfologías; se destacan los poliédricos parciales (27\%), una pieza bipolar (Figura 5B) y un nódulo testeado. En cuanto a la cantidad de lascados, los que presentan entre 2 y 4 extracciones representan el $45 \%$, mientras que los que muestran entre cinco y 11 son el $55 \%$. Se destaca la pieza $\mathrm{N}^{\circ} 11$ que se encuentra en estado activo y presenta 11 lascados (Figura 5C).

Finalmente, el único instrumento identificado (Figura 5D) se registró en la E2 y corresponde a un filo natural con rastros complementarios. La pieza presenta un filo recto, largo, de arista regular, bisel asimétrico de $48^{\circ}$ y posee microrretoques de uso y aristas alisadas. Estas características sugieren que la pieza fue utilizada para acciones de raspado sobre materiales resistentes (i.e. madera, hueso) (Aschero 1975, 1983; Pautassi 2010).
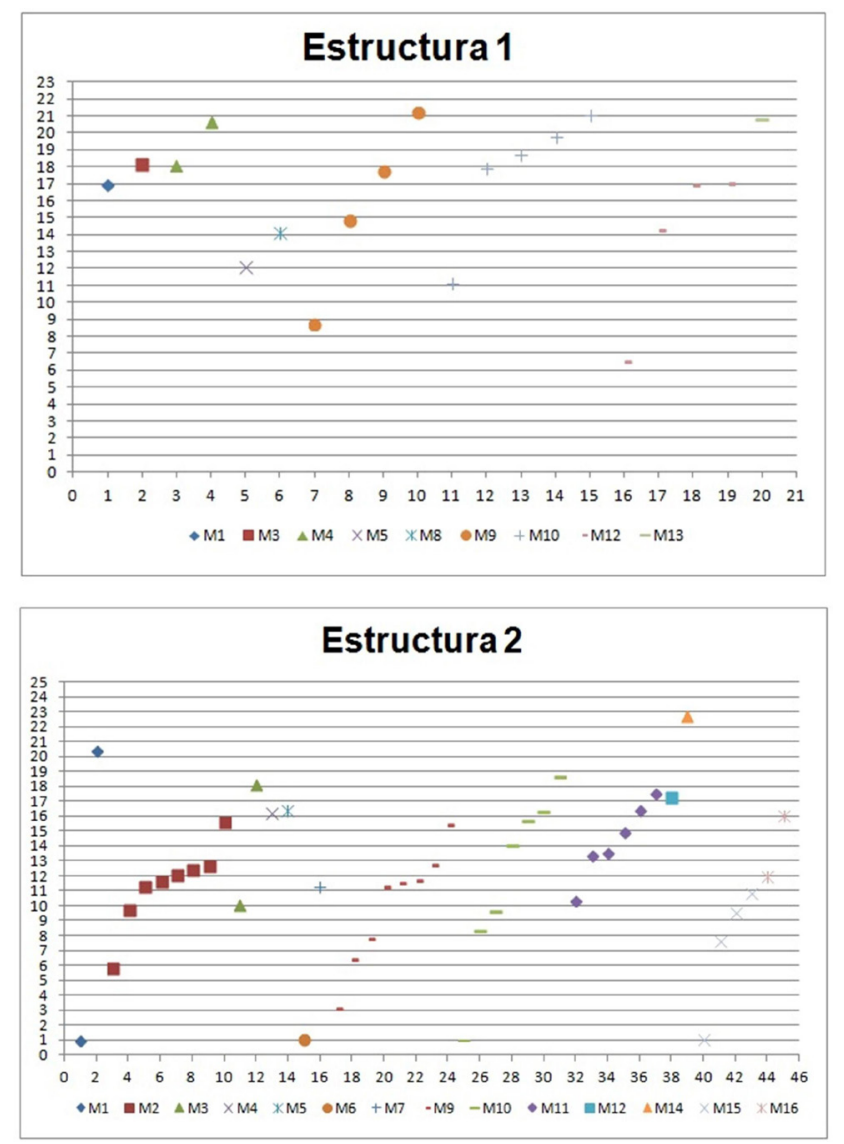

Figura 4. Gráfico de dispersión del análisis No Tipológico de E1 (arriba) y E2 (abajo). Cada conjunto con la misma referencia representa los desechos de talla correspondientes a un mismo nódulo mínimo y cada referencia es un evento de extracción que sigue el orden dado por el eje Y.

Figure 4. Dispersion graph of the Non-typological analysis of E1 (up) and E2 (down). Each set with the same reference represents the knapping wastes corresponding to the same minimum nodule and each reference is an extraction event that follows the order given by the $Y$ axis.

\begin{tabular}{|c|c|c|}
\hline Estructura & MANA & Promedio \\
\hline \multirow{9}{*}{1} & M1 & 16,95 \\
\hline & M3 & 18,16 \\
\hline & M4 & 19,41 \\
\hline & M5 & 12,05 \\
\hline & M8 & 14,11 \\
\hline & M9 & 15,67 \\
\hline & M11 & 17,74 \\
\hline & M12 & 13,73 \\
\hline & M13 & 20,80 \\
\hline \multirow{14}{*}{2} & M1 & 21,37 \\
\hline & M2 & 11,38 \\
\hline & M3 & 14,10 \\
\hline & M4 & 16,18 \\
\hline & M5 & 16,36 \\
\hline & M6 & 1,00 \\
\hline & M7 & 11,25 \\
\hline & M9 & 10,40 \\
\hline & M10 & 11,96 \\
\hline & M11 & 14,39 \\
\hline & M12 & 17,20 \\
\hline & M14 & 22,69 \\
\hline & M15 & 7,24 \\
\hline & M16 & 13,97 \\
\hline
\end{tabular}

Tabla 2. Promedio de momento de extracción para los eventos de talla de E1 y E2.

Table 2. Average moment of extraction for the knapping wastes of $E 1$ and $E 2$.

\section{Discusión}

El predominio en los conjuntos de las categorías de desechos de talla y núcleos, de los nódulos múltiples y de las extracciones centradas entre los momentos seis al 22 permite plantear que la producción lítica desarrollada en E1 y E2 se habría vinculado con las actividades de talla más tempranas, apuntando a la obtención y reducción de formas bases. Las evidencias del predominio de las lascas internas, sin corteza y con talones preparados, así como de los núcleos agotados o parcialmente agotados, sin corteza y con cinco a 11 lascados, parecen vincularse con actividades de preparación de formas bases para su formatización. El alto grado de fragmentación de los desechos, las escasas lascas bipolares y talones puntiformes, y el único núcleo bipolar, parece vincularse con técnicas tendientes a obtener rápidamente formas base.

Por su parte, los escasos nódulos mínimos (M.A.N.A.) simples, las pocas extracciones inferiores al momento seis, los bajos porcentajes de lascas externas y talones corticales, junto a los núcleos con dos o cuatro lascados, parecen relacionarse con el testeo de nódulos y el descortezamiento de núcleos, cuya recurrencia parece haber sido menor en el marco de estas tareas iniciales de la producción lítica. También es posible pensar, debido al registro de núcleos parcialmente agotados y activos, en un almacenamiento de materias primas.

Las evidencias apuntan a que hacia el interior de E1 y 
Tabla 3. Resultados del análisis tecno-tipológico de los desechos de talla de E1 y E2.

Table 3. Results of the techno-typological analysis of knapping wastes of E1 and E2.

\begin{tabular}{|l|c|c|c|}
\hline \multicolumn{1}{|c|}{ Variable } & E1 & E2 & Total \\
\hline Origen de la Extraccion & \multicolumn{3}{|c|}{} \\
\hline Lasca Interna & 64 & 158 & 222 \\
\hline Lasca Externa & 7 & 12 & 19 \\
\hline Lasca Bipolar & 2 & 3 & 5 \\
\hline Sub Total & 73 & 173 & 246 \\
\hline Tamaño & \multicolumn{5}{|c|}{} \\
\hline Pequeño & 1 & 13 & 14 \\
\hline Mediano Pequeño & 7 & 18 & 25 \\
\hline Mediano Grande & 7 & 6 & 13 \\
\hline Grande & 3 & 8 & 11 \\
\hline Muy Grande & 2 & 0 & 2 \\
\hline Sub Total & 20 & 45 & 65 \\
\hline Corteza & \multicolumn{5}{|c|}{} \\
\hline 0\% & 14 & 28 & 42 \\
\hline 1-25\% & 4 & 10 & 14 \\
\hline 26-50\% & 2 & 7 & 9 \\
\hline Sub Total & 20 & 45 & 65 \\
\hline Tipo de talón & \multicolumn{5}{|c|}{} \\
\hline Cortical & 17 & 21 & 38 \\
\hline Liso & 35 & 104 & 139 \\
\hline Diedro & 2 & 1 & 3 \\
\hline Facetado & 0 & 1 & 1 \\
\hline Puntiforme & 3 & 3 & 6 \\
\hline Sub Total & 57 & 130 & 187 \\
\hline & \multicolumn{4}{|c|}{} \\
\hline
\end{tabular}

E2 predominaron las etapas iniciales de la secuencia productiva del cuarzo, tales como el testeo de nódulos, el descortezamiento de núcleos, la extracción de formas base y la reducción/preparación de lascas; aunque se habría dado un mayor énfasis de estas dos últimas actividades. Por lo tanto, en las estructuras se habrían producido los momentos tempranos de la historia de vida (sensu Schiffer 1972) de los artefactos de cuarzo, obteniéndose formas bases aptas para la manufactura y uso de instrumentos que habrían continuado su trayectoria en otros espacios.

Al vincular estas actividades con el paisaje socialmente construido en que se enmarcan las estructuras, se puede avanzar en la comprensión de las prácticas en las que el cuarzo y las personas se relacionaron. Por un lado, a dos $\mathrm{km}$ de las estructuras, sobre diferentes sectores del cerro Ampuqcatao, se ubican canteras de cuarzo que presentan evidencias de extracciones persistentes anteriores y posteriores al segundo milenio D.C. (Manasse 2011). A su vez, el tamaño de las estructuras (que solo permitirían la ocupación de un número reducido de personas), la presencia de escasos fragmentos cerámicos, el registro de un filo natural asociable a tareas de raspado, y la ubicación de E1 y E2 en sectores con alta visibilidad de las terrazas de cultivo y del área circundante, permiten
Tabla 4. Núcleos registrados en E1 y E2.

Table 4. Cores registered in E1 and E2.

\begin{tabular}{|l|l|c|c|l|}
\multicolumn{1}{|c|}{ Estado } & Corteza & Lascados & \multicolumn{1}{c|}{ Morfología } \\
\hline Parcialmente agotado & $0 \%$ & 5 & Poliédrico parcial \\
\hline Fracturado & & 5 & Indet. por fractura \\
\hline Agotado & $60 \%$ & 2 & Nódulo testeado \\
\hline Agotado & $0 \%$ & 3 & Piramidal irregular \\
\hline Fracturado & & 3 & Indet. por fractura \\
\hline Agotado & $15 \%$ & 2 & Pseudo piramidal \\
\hline Parcialmente agotado & $0 \%$ & 4 & Prismático irregular \\
\hline Parcialmente agotado & $0 \%$ & 9 & Poliédrico parcial \\
\hline Parcialmente agotado & $0 \%$ & 7 & Poliédrico parcial \\
\hline Agotado & $15 \%$ & 7 & Bipolar \\
\hline Activo & $10 \%$ & 11 & Pseudo prismático \\
\hline
\end{tabular}

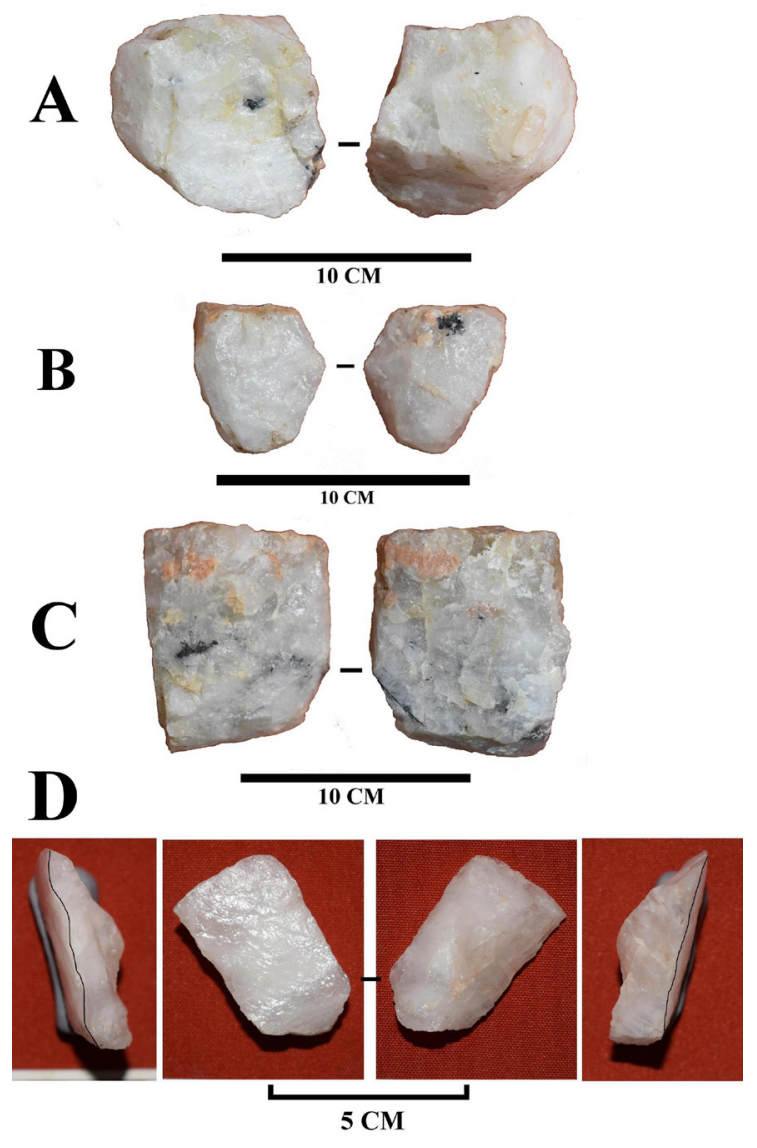

Figura 5. Artefactos de El Alto. A: Núcleos 1 (parcialmente agotado). B: Núcleo 10 (núcleo bipolar). C: Núcleo 11 (activo). D: filo natural con rastros complementarios.

Figure 6. Artefacts from El Alto site. A: Core 1 (partly spent). B: Core 10 (bipolar). C: Core 11 (active); D: natural edge with complementary traces.

suponer un uso de los recintos de manera temporaria en el marco de actividades vinculadas quizás con el control de la producción de alimentos.

Se plantea la posibilidad que las personas y los 
artefactos de cuarzo se podrían haber vinculado en el marco de estrategias embedded (Binford 1979) relacionadas con prácticas de producción agropastoril. De esta manera, las personas implicadas en el uso de estos materiales cuarzosos podrían haber realizado actividades iniciales de talla (i.e. obtención de materia prima, testeo y descortezamiento de núcleos, obtención/preparación de formas base) integradas con las tareas productivas (i.e. siembra, cosecha, pastoreo), conformando en el sector norte del cerro Ampuqcatao verdaderos taskscapes (Ingold 1993), al corporizar en las estructuras y materiales el paisaje habitado y vivido por los agentes.

Este tipo configuración de paisajes ha sido identificado en otros sectores del valle durante el primer y segundo milenio D.C. Franco Salvi y Molar (2018) indican la presencia de una estructura excepcional en el sitio La Bolsa 2, la cual fue datada en 1286-1392 D.C. y que muestra una instalación agrícola con un "alerorefugio" de pequeñas dimensiones y materiales líticos y cerámicos asociables a ocupaciones temporarias. Su interpretación de este contexto arqueológico apunta al uso del alero-refugio como lugar de control de las actividades productivas, separado de las ocupaciones residenciales. Por otra parte, Berberián (1994) excavó en el sitio Casas Viejas un pequeño recinto en un despedre de cultivo del primer milenio D.C., cercano a un montículo de uso ritual, en el cual se identificaron materiales relacionados con acciones de fumar, tejer, y preparar y consumir alimentos. Todo esto lleva a pensar en un uso temporario del recinto durante las labores agrícolas.

Es posible que las estructuras de El Alto, La Bolsa 2 y Casas Viejas compartan un uso temporario de las mismas durante períodos especiales de los ciclos productivos, instalándose en ellas algunas personas sólo durante los momentos de mayor demanda de trabajo (Manasse 2011). En el caso particular de El Alto, la cercanía de las fuentes de cuarzo pudo permitir que en los momentos de descanso y ocio del trabajo agrícolapastoril los ocupantes se dedicaran a la reducción de nódulos y núcleos para obtener piezas transportables a espacios residenciales, así como a la acumulación de materias primas, a modo de cache (Binford 1979), previendo un retorno a las estructuras. En este sentido, contrastan las actividades y artefactos líticos registrados en espacios habitados diariamente del valle de Tafí, donde predominan las etapas intermedias y finales de producción de artefactos, así como el uso, mantenimiento y descarte de instrumentos (Franco Salvi et al. 2016). Todas estas actividades (construir estructuras, producir alimentos, tallar, descansar, alimentarse, fumar, tejer) realizadas por las personas son las que conformaron el paisaje que estudiamos actualmente.

\section{Conclusiones}

A partir de los análisis efectuados se pudo reconocer qué etapas de la secuencia productiva lítica del cuarzo se desarrollaron en las estructuras E1 y E2. En este aspecto, los métodos M.A.N.A y No Tipológico, así como su complementación con estudios tecno-tipológicos, permitieron identificar principalmente las etapas iniciales de la secuencia. Por otra parte, la vinculación de estos resultados y del paisaje natural y social en que se desarrollan las tareas líticas permitió comprender las prácticas que habrían relacionado materiales, personas y paisaje, de modo que observamos el posible desarrollo de tareas de talla enmarcadas en estrategias embedded vinculadas a actividades de producción agropastoril. Es decir, las estructuras podrían haber funcionado como pequeños talleres líticos de uso temporario.

Por otra parte, el estudio permitió determinar la potencialidad de las metodologías M.A.N.A y No Tipológica para el estudio de artefactos de cuarzo. Aquí se observó que estas herramientas complementan los análisis tecno-tipológicos, aumentando el potencial explicativo respecto de las actividades de talla lítica presentes en el registro arqueológico. Los métodos permiten abordar el estudio de los conjuntos de cuarzo a nivel de evento de talla, logrando definir y caracterizar eventos tan discretos como el descortezamiento de un núcleo o la formatización de un instrumento. Es necesario recalcar que los métodos presentan una desventaja importante al considerar en su análisis únicamente las lascas enteras, ya que son las que contienen las variables necesarias para los modelos aplicados, de manera que reducen la muestra analizada, sobre todo en conjuntos de cuarzos donde la fragmentación suele ser alta. Sin embargo, como ya se mencionó, su complementariedad con estudios tecno tipológicos, permiten que su uso aumente la mirada sobre las prácticas de tallado del cuarzo. Por último, la utilización de estas metodologías correlacionadas con otros contextos, como canteras y áreas residenciales, tiene el potencial de brindar información sobre las estrategias tecnológicas implementadas, los modos de producción, técnicas de talla, entre otras.

Córdoba, 15 de Abril 2019

\section{Agradecimientos}

A la Comunidad Indígena del Valle de Tafí con quienes se realizaron los trabajos de campo en el cerro Ampuqcatao buscando revalorizar el patrimonio cultural originario. A los comuneros Mónica Mendez y Fabián Lara por su hospitalidad y colaboración en todas las tareas. A los miembros del Equipo de Arqueología del Sur de las Cumbres Calchaquíes: J. Salazar, V. Franco Salvi, R. Molar, F. Franco, G. Moyano, A. Vazquez Fiorani, S. 
Chiavassa Arias y D. Carrasco. Los trabajos de campo y de laboratorio se hicieron en el marco del permiso otorgado por la Dirección de Patrimonio de la provincia de Tucumán. Los trabajos y análisis se realizaron en el marco de la Beca BITS otorgada por la SECyT-UNC, y con el financiamiento de Koeki Zaidan Hojin Toyota Zaidan (公益財団法人トヨタ財団) The Toyota Foundation [TYTID: D16-R-0718].

\section{Bibliografía}

Aschero, C. (1975) Ensayo para una clasificación morfológica de los artefactos líticos aplicada a estudios tipológicos comparativos. Informe inédito presentado al CONICET.

Aschero, C. (1983). Ensayo para una clasificación morfológica de artefactos líticos aplicada a estudios tipológicos comparativos. Revisión. Cátedra de Ergología y Tecnología. Facultad de Filosofía y Letras, Universidad de Buenos Aires.

Berberián, E. (1994). Sistemas de asentamiento en el Valle de Tafí. Informe elevado a CONICET.

Berberián, E. E. y A. E. Nielsen. (1988). Sistemas de Asentamientos Prehispánicos en el Valle de Tafí. Editorial Comechingonia. Córdoba.

Binford, L. R. (1979). Organization and formation processes: looking at curated technologies. Journal of Anthropological Research 35:255-273.

Cattáneo, G. R. (1994). Estrategias tecnológicas: un modelo aplicado a las ocupaciones prehistóricas del Valle de Copacabana, N.O. de la Provincia de Córdoba. Publicaciones de Arqueología 47: 1-30.

Cattáneo, G. R. (2002). Una Aproximación a la Organización de la Tecnología Lítica entre los CazadoresRecolectores del Holoceno Medio/Pleistoceno Final en la Patagonia Austral, Argentina. Tesis de Doctorado. Universidad Nacional de la Plata.

Flegenheimer, N. y R. Cattáneo (2013). Análisis comparativo de desechos de talla en contextos del Pleistoceno Final/Holoceno Temprano de Chile y Argentina. Magallania 41(1): 171-192.

Franco Salvi, V. y R. M. Molar (2018). Paisajes agrarios del segundo milenio de la era en el sector norte del Valle de Tafí (Tucumán, Argentina). Estudios Atacameños. Arqueologia y Antropologia Sudamericanas 57: 45-63.

Franco Salvi, V., J. Salazar y J. Montegú (2016). Prácticas cotidianas y vida aldeana. Un análisis desde la tecnología lítica en el Valle de Tafí (Tucumán, Argentina). Cuadernos dels Instituto Nacional de Antropología y Pensamiento Latinoamericano 25 (2): 141-158.

Frison, G. (1974). The Casper Site. A Hell Gap Bison Kill on the High Plains. New York: academic Press.

González, A. R. y V. Núñez Regueiro (1960). Preliminary Report on Archaeological Research in Tafí Valley, NW Argentina. Actas del $34^{\circ}$ Congreso de Americanistas, pp 18-25. Viena.

Ingbar, E., M. Larson y B. Bradley (1989). A non typological approach to débitage analysis. En Experiments in lithic technology, editado por D. Amick y R. Mauldin, pp. 6799. Oxford: Archaeopress (BAR S528).

Ingold, T. (1993). The temporality of the landscapes. World Archaeology: Conceptions of Time and Ancient Society 25: 189-208.

Kelly, R. L. (1985). Hunter-Gatherer Mobility and Sedentism: A Great Basin Study. Tesis Doctoral, University of Michigan.

Kulemeyer, J.A. y L.R. Laguna (1995). La representación gráfica de artefactos líticos arqueológicos. En Kulemeyer, J.A. Arqueología. Algunas cuestiones relativas a la recolección y presentación de hallazgos. Jujuy: CICNA, serie Cultura y naturaleza andinas, documento número 1.

Larson, M. L. y M. Kornfeld (1997). Chipped stone nodules: theory, method, and examples. Lithic Technology 22: 4-18.

Manasse, B. (2011). Arqueología en el borde andino del Noroeste Argentino: sociedades del último milenio en el Valle de Tafí, Prov. de Tucumán-Rep. Argentina. Tesis doctoral. Facultad de Ciencias Naturales, Universidad Nacional de la Plata.

Núñez Regueiro, V. (1974). Conceptos instrumentales y marco Teórico en relación al análisis del desarrollo Cultural del Noroeste Argentino. Revista del Instituto de Antropología de Córdoba 5: 169-190.

Orquera, L.A. y E. L. Piana (1986). Normas para la descripción de objetos arqueológicos de piedra tallada. Publicación Especial 1. CADIC. Centro Austral de Investigaciones Científicas, Ushuaia, Argentina.

Pautassi, E. A. (2014). La talla y uso del cuarzo, una aproximación metodológica para la comprensión de cazadores-recolectores de Córdoba. Tesis Doctoral inédita. Facultad de Filosofía y Humanidades, Universidad Nacional de Córdoba.

Salazar, J. (2010). Reproducción social doméstica y 
asentamientos residenciales entre el 200 y el 800 d.C. en el valle de Tafí, Tucumán. Tesis Doctoral. Facultad de Filosofía y Humanidades, Universidad Nacional de Córdoba.

Salazar, J. (2014). Las ocupaciones originarias en el sector norte del Cerro Pelao (Ampuqcatao). Informe arqueológico. Universidad Nacional de Córdoba.

Salazar, J, V. Franco Salvi, E. Berberián y S. Clavero (2008). Contextos domésticos del Valle de Tafí, Tucumán, Argentina (200-1000 AD). Werken 10: 25-48. Chile.

Sario, G. (2011). Poblamiento humano en la provincia de San Luis: una perspectiva arqueológica a través del caso de la organización de la tecnología en Estancia La Suiza. Tesis doctoral. Facultad de Filosofía y Humanidades, Universidad Nacional de Córdoba.
Scattolin, M. C. (2006). De las Comunidades aldeanas a los Curacazgos en el Noroeste Argentino. Boletín de Arqueología PUCP 10: 357-398.

Schiffer, M. (1972). Archaelogical context and systemic context. American Antiquity 37(2): 156-165.

Servicio Geológico Argentino (2010). Carta Geológica 2766-II San Miguel de Tucumán.

Sullivan, A. y K. C. Rozen (1985). Debitage analysis and archaeological interpretation. American Antiquity 50(4):755-779.

Williams, V., P. Villegas, M. S. Gheggi, y M. G. Chaparro (2005). Hospitalidad e intercambio en los valles mesotermales del Noroeste Argentino. Boletín de Arqueología PUCP 9: 335-372. 